\title{
New probes SWIRling around
}

A new set of short-wavelength infrared (SWIR) quantum dot probes for improved deep tissue in vivo optical imaging.

Non-invasive imaging in living animals is a critical tool for scientists to understand biological mechanisms and disease progression. Improvements in fluorescent probes have been especially important for in vivo imaging, adding new tools that enable scientists to label and track specific molecules. But fluorescent probes still have many drawbacks that limit their use for imaging within intact animals. Autofluorescence and scattering of light decrease contrast

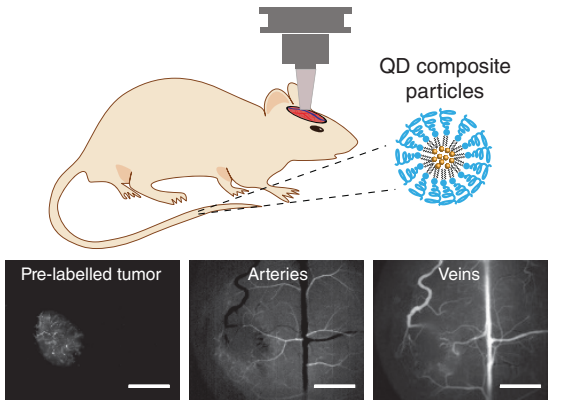

Quantum dot SWIR imaging in the mouse brain.

and spatial resolution; problems that only get worse the deeper scientists try to image.
In a new paper published in Nature Biomedical Engineering, Moungi Bawendi and colleagues introduce a set of quantum dots that dramatically improve imaging in the short-wavelength infrared region (SWIR) (Nat. Biomed. Eng. 1, 0056; 2017). Using mice, they demonstrate the power of multicolor SWIR imaging by tracking lipoprotein turnover in various tissue types simultaneously, heartbeat and breathing in awake and unrestrained animals, and generating detailed flow maps of brain vasculature.

Dustin M. Graham 